\title{
ESR Fine Structure of Manganese Ions in Zeolite A Detects Strong Variations of the Coordination Environment
}

\author{
Dirk E. De Vos, ${ }^{\dagger}$ Bert M. Weckhuysen,, and Thomas Bein $*, \dagger$ \\ Contribution from the Department of Chemistry, Purdue University, \\ West Lafayette, Indiana 47907, and Centrum voor Oppervlaktechemie en Katalyse, \\ K. U. Leuven, Kardinaal Mercierlaan 92, 3001 Heverlee, Belgium
}

Received January 2, $1996^{\otimes}$

\begin{abstract}
The electron spin resonance spectra of $\mathrm{Mn}^{2+}$ exchanged zeolite A have been investigated as a function of the monovalent co-cation $\left(\mathrm{K}^{+}, \mathrm{Na}^{+}, \mathrm{Li}^{+}, \mathrm{Cs}^{+}\right.$, or $\left.\mathrm{NH}_{4}{ }^{+}\right), \mathrm{Mn}^{2+}$ content, recording frequency, and temperature. Three new $\mathrm{Mn}^{2+}$ species are observed with a well-resolved fine structure; this allows for the first time a direct quantitative determination of the zero-field splitting (ZFS) parameters in zeolites. In hydrated zeolites, three ESR active Mn ${ }^{2+}$ species are observed, characterized by different values for the ZFS parameter $D$. Species $\mathbf{I}$ has $D=0.035 \mathrm{~cm}^{-1}$. Species II is closer to a regular octahedron, with $D=0.010 \mathrm{~cm}^{-1}$. Species III, with $D=0.14 \mathrm{~cm}^{-1}$, is in a strongly axially distorted coordination. Species I is dominant in MnKA, MnCsA, and $\mathrm{MnNH}_{4} \mathrm{~A}$, while II and III are found in $\mathrm{MnNaA}$ and MnLiA. In fully dehydrated zeolites, two species are observed. Species IV has a small hyperfine constant $A$ and is present in dry NaA and KA. Species $\mathbf{V}$ is observed in dry LiA; it has axial symmetry with a large, temperature-dependent $D$. Species $\mathbf{V}$ probably represents $\mathrm{Mn}^{2+}$ in a 3-fold coordination in a 6-ring. In partially hydrated zeolites, a tetrahedral species VI is observed. The spectroscopic data elucidate the location of manganese(II) ions in zeolite A, particularly at relatively low metal loadings.
\end{abstract}

\section{Introduction}

The ESR fine structure of transition metal ions with several unpaired electrons, such as high-spin $\mathrm{Mn}^{2+}, \mathrm{Fe}^{3+}$, and $\mathrm{Cr}^{3+}$, is determined by the splitting of the energy levels in zero field. Analysis of the zero-field splitting (ZFS) parameters $D$ and $E$ provides direct insight into the symmetry and coordination of these ions. Especially for $\mathrm{Mn}^{2+}$, where common techniques such as electronic spectroscopy and magnetic measurements yield little useful information, the analysis of the ZFS parameters based on the ESR spectra has been extremely helpful in elucidating the structure of numerous $\mathrm{Mn}^{2+}$ compounds. ${ }^{1}$

In the study of the coordination and siting of transition metal ions in zeolites and related molecular sieves, ESR has been a major tool. ${ }^{2}$ However, most studies have concentrated on intrazeolitic $\mathrm{d}^{1}$ or $\mathrm{d}^{9}$ metal ions, where structural information is obtained primarily from anisotropy in the $\boldsymbol{g}$ or $\boldsymbol{A}$ tensor. For ions with higher spin multiplicity, knowledge of the ZFS parameters is essential for structural understanding. However, in the case of $\mathrm{Fe}^{3+}$ and $\mathrm{Cr}^{3+}$, the typical zeolite spectra do not display sufficient resolution for quantitative determination of $D$ and $E .^{3,4}$ In a recent detailed study, Goldfarb et al. discuss the role of ZFS in the spectrum of iron-containing zeolites. ${ }^{4}$ One usually observes the limiting situations $g_{\text {eff }}=2$ (cubic symmetry, $D$ and $E \ll h v$ ) and $g_{\text {eff }}=4.3$ (rhombic symmetry, $D \gg h v, E / D$ close to $1 / 3$ ).

\footnotetext{
* Author to whom correspondence should be addressed.

$\dagger$ Purdue University.

$¥$ Centrum voor Oppervlaktechemie en Katalyse.

${ }^{\otimes}$ Abstract published in Advance ACS Abstracts, August 1, 1996.

(1) (a) Dowsing, R. D.; Gibson, J. F.; Goodgame, D. M. L.; Goodgame, M.; Hayward, P. J. Nature 1968, 219, 1037. (b) Goodgame, M.; Okey, J. N. J. Chem. Soc., Dalton Trans. 1985, 75.

(2) (a) Kevan, L. Acc. Chem. Res. 1987, 20, 1. (b) Kevan, L. Electron Spin Resonance, A Specialist Periodical Report 1991, 12B, 99. (c)
} Schoonheydt, R. A. Catal. Rev. Sci. Eng. 1993, 35, 129.

(3) Pearce, J. R.; Sherwood, D. E.; Hall, M. B.; Lunsford, J. H. J. Phys. Chem. 1980, 84, 3215.

(4) Goldfarb, D.; Bernardo, M.; Strohmaier, K. G.; Vaughan, D. E. W.; Thomann, H. J. Am. Chem. Soc. 1994, 116, 6344.
In the specific case of $\mathrm{Mn}^{2+}$ molecular sieves, earlier ESR studies have mainly been concerned with $\mathrm{X}$ or $\mathrm{Y}$ zeolites. 5,6 Variations of the co-cation population and hydration of these zeolites influence the ESR spectra, and at least five different $\mathrm{Mn}^{2+}$ species have been proposed in $\mathrm{Y}$ zeolites alone. In addition, the spectra of Mn-substituted (silico)aluminophosphate or mesoporous molecular sieves have been reported and discussed. ${ }^{7}$ A common characteristic of all reported spectra of $\mathrm{Mn}^{2+}$ in molecular sieves is that only the central $M_{S}=+1 / 2 \leftrightarrow$ $-1 / 2$ transition is resolved; the non-central fine lines are typically broadened, making it difficult to determine $D$ and $E$. Nevertheless, knowledge of the ZFS parameters is important, as any departure from a cubic octahedral or tetrahedral symmetry is expressed primarily by changes of the ZFS parameters.

In this work, we report for the first time on the direct observation of the fine structure in the ESR spectra of $\mathrm{Mn}^{2+}$. exchanged A zeolites. The X- and Q-band ESR spectra of $\mathrm{Mn}^{2+}$ in this zeolite are strongly influenced by the nature of the cocation $\left(\mathrm{Li}^{+}, \mathrm{Na}^{+}, \mathrm{K}^{+}, \mathrm{Cs}^{+}\right.$, and $\left.\mathrm{NH}_{4}^{+}\right)$and by the hydration state of the molecular sieve. Attention is directed primarily toward the identification of the spectral features. Apart from three $\mathrm{Mn}^{2+}$ species with small ZFS, previously reported for zeolite $\mathrm{A}$, we describe three new $\mathrm{Mn}^{2+}$ species with wellobservable fine-splitting patterns. From these, reliable values for the fine-splitting parameter $D$ are derived. These data are

(5) (a) Barry, T. I.; Lay, L. A. Nature 1965, 208, 1312. (b) Barry, T. I.; Lay, L. A. J. Phys. Chem. Solids 1966, 27, 1821. (c) Barry, T. I.; Lay, L. A. J. Phys. Chem. Solids 1968, 29, 1395.

(6) (a) Tikhomirova, N. N.; Nikolaeva, I. V.; Demkin, V. V.; Rosolovskaya, E. N.; Topchieva, K. V. J. Catal. 1973, 29, 105. (b) Tikhomirova N. N.; Nikolaeva, I. V.; Demkin, V. V.; Rosolovskaya, E. N.; Topchieva, K. V. J. Catal. 1973, 29, 500. (c) Tikhomirova, N. N.; Nikolaeva, I. V.; Rosolovskaya, E. N., Demkin, V. V.; Topchieva, K. V. J. Catal. 1975, 40, 61. (d) Tikhomirova, N. N.; Nikolaeva, I. V. J. Catal. 1975, 40, 135. (e) Tikhomirova, N. N.; Nikolaeva, I. V. Zh. Fiz. Khim. 1981, 55, 2224.

(7) (a) Lee, C. W.; Chen, X.; Brouet, G.; Kevan, L. J. Phys. Chem. 1992, 96, 3110. (b) Brouet, G.; Chen, X.; Lee, C. W.; Kevan, L. J. Am. Chem. Soc. 1992, 114, 3720. (c) Levi, Z.; Raitsimring, A. M.; Goldfarb, D. J. Phys. Chem. 1991, 95, 7830. (d) Zhao, D.; Goldfarb, D. J. Chem. Soc., Chem. Commun. 1995, 875. 
compared to spectroscopic data for other cations, and the implications for the distribution of $\mathrm{Mn}^{2+}$ over different cation sites in zeolite A are discussed.

\section{Experimental Section}

KA (from Alfa) and NaA zeolites (from PQ) were used in this work. $\mathrm{LiA}$ was obtained by stirring $\mathrm{NaA}$ three times in a 5 -fold $\mathrm{LiCl}$ excess at $333 \mathrm{~K}$ for $6 \mathrm{~h}$, followed by extensive washing with deionized water. An analogous procedure, using a 3-fold excess of $\mathrm{CsCl}$ or $\mathrm{NH}_{4} \mathrm{Cl}$, was applied to obtain $\mathrm{CsNaA}, \mathrm{CsKA}$, and $\mathrm{NH}_{4} \mathrm{~A}$ zeolites. $\mathrm{Mn}^{2+}$ exchanges were performed for a minimum of $3 \mathrm{~h}$ at room temperature, by stirring the hydrated zeolite in dilute solutions of $\mathrm{MnCl}_{2} \cdot 4 \mathrm{H}_{2} \mathrm{O}$ (Mallinckrodt). A minimum volume to zeolite weight ratio of $100 \mathrm{~mL}$ per $\mathrm{g}$ and a maximum $\mathrm{Mn}$ concentration of $2 \mathrm{mM}$ were used. The $\mathrm{pH}$ during exchange was kept between 6 and 7 by addition of dilute $\mathrm{HCl}$ to prevent the base-promoted oxidation of $\mathrm{Mn}^{2+}$ to $\mathrm{Mn}^{3+} .8$ The resulting white suspensions were filtered and washed with copious amounts of deionized water. A high crystallinity of the $\mathrm{Mn}^{2+}$-exchanged zeolites was confirmed by X-ray diffraction using a XDS 2000 diffractometer of Scintag. The Mn contents were 0.025, 0.1, 0.4, 1.7, and $10.0 \mathrm{wt} \%$. Zeolites were dehydrated by heating under a flow of oxygen at a rate of $1 \mathrm{~K}$ per min up to $673 \mathrm{~K}$; this temperature was maintained for $6 \mathrm{~h}$. Rehydration was performed by exposure of the dehydrated zeolites at room temperature to a relative humidity of $79 \%$ over a saturated $\mathrm{NH}_{4}$ $\mathrm{Cl}$ solution.

X-band ESR spectra of the Mn zeolite samples were recorded with a Bruker ESP-300 apparatus between 7 and $400 \mathrm{~K}$. Q-band ESR spectra were obtained with a Varian spectrometer with cooling accessory. ESR spectral simulations were performed with the Q-Pow simulation program of Dr. M. Nilges (University of Illinois at Urbana-Champaign). Magnetic susceptibility measurements were performed using a MPMS SQUID magnetometer in a field of $0.1 \mathrm{~T}$.

\section{Results}

Samples with a $\mathrm{Mn}^{2+}$ content between 0.025 and 0.4 wt $\%$ have ESR spectra with optimum signal-to-noise ratios, whereas higher Mn loadings resulted in a serious loss of spectral resolution, presumably due to dipolar interactions. At $10 \mathrm{wt}$ $\% \mathrm{Mn}$, there is only one, structureless absorption.

ESR fine-splitting patterns may arise not only from monomeric species but also from species with more nuclei; for example, fine structure is well documented for $\mathrm{Mn}-\mathrm{Mn}$ pairs in $\mathrm{CaO}$, or for $\mathrm{Cu}-\mathrm{Cu}$ pairs in some $\mathrm{Y}$ zeolites. ${ }^{9}$ In the absence of hyperfine structure, occurrence of $\mathrm{Mn}$ pairs or larger aggregates may thus be difficult to exclude. Therefore MnKA, $\mathrm{MnLiA}$, and $\mathrm{MnNaA}(0.4$ wt \% Mn) were subjected to SQUID magnetization measurements at constant field $(0.1 \mathrm{~T})$ between 10 and $200 \mathrm{~K}$. In this temperature range, the $\mathrm{Mn}$ in these zeolites displayed essentially identical Curie behavior, even if the ESR spectra varied considerably. Therefore it seems that fine structure in the ESR spectra is due to different coordination geometries of mononuclear $\mathrm{Mn}$ species, rather than to the presence of Mn aggregates.

The line identification for $\mathrm{Mn}^{2+}$ ESR powder spectra is based on the evaluation of the spin Hamiltonian developed by Abragam and Bleaney. ${ }^{10}$ In the case of axial symmetry, the zero-field splitting is described by the parameter $D$. For $\mathrm{Mn}^{2+}$, with $S=5 / 2$, this results in a splitting of the resonance into five fine transitions $\left(M_{S}=+1 / 2 \leftrightarrow-1 / 2 ; M_{S}= \pm^{3 / 2} \leftrightarrow \pm^{1 / 2} ; M_{S}=\right.$ $\pm 5 / 2 \leftrightarrow \pm 3 / 2$ ). In powder spectra, the position of these lines is

(8) Baes, C. F.; Mesmer, R. E. The Hydrolysis of Cations; Wiley: New York, 1976; p 220.

(9) (a) Harris, E. A. J. Phys. C: Solid State Phys. 1972, 5, 338. (b) Chao, C. C.; Lunsford, J. H. J. Chem. Phys. 1972, 57, 2890.

(10) (a) Bleaney, B.; Ingram, D. J. E. Proc. R. Soc. (London) 1951, A205, 336. (b) Bleaney, B.; Rubins, R. S. Proc. Phys. Soc. 1961, 77, 103; 1961, 78,778 (corrigendum). a function of $\theta$, the angle between the local axis of symmetry and the external magnetic field. The most intense lines in the spectrum correspond to the $\theta=90^{\circ}$ maxima of the five $\Delta M_{S}$ $= \pm 1$ transitions, which are separated in first order by $D .{ }^{11}$ The absorption at other angles tends to be weak, with the exception of the $\theta=41.8^{\circ}$ orientation, where the central $M_{S}=+1 / 2 \leftrightarrow$ $-1 / 2$ transition has another intensity maximum. Each of these six lines is further split into a sextet by the interaction with the Mn nuclear spin $I=5 / 2$. Apart from these allowed $\Delta M_{S}=$ $\pm 1, \Delta M_{I}=0$ lines, forbidden $\Delta M_{S}= \pm 1, \Delta M_{I}= \pm 1$ transitions are often observed, and they gain intensity at lower measurement frequency. ${ }^{10 \mathrm{~b}}$ Typically, they give rise to five doublets between the six hyperfine components of the allowed $M_{S}= \pm 1 / 2 \leftrightarrow-1 / 2$ transition. Finally, around half field, the forbidden $\Delta M_{S}= \pm 2$ transitions may be observed.

Several criteria can be used to determine the magnitude of $D$ from powder spectra. The most obvious method consists in the evaluation of the positions of the $\theta=90^{\circ}$ absorption maxima of the allowed $\Delta M_{S}= \pm 1, \Delta M_{I}=0$ transitions, which are roughly spaced by $D$. However, due to their angle dependence, the four outer transitions may be smeared out beyond resolution. Secondly, one may use the (relative) positions of the $\theta=90^{\circ}$ and $41.8^{\circ}$ absorption maxima of the central $M_{S}=+1 / 2 \leftrightarrow-1 / 2$ fine line. ${ }^{12,13}$ These are subject to small shifts, proportional to $D^{2} / H_{0}$, downfield $\left(\theta=90^{\circ}\right)$ or upfield $\left(\theta=41.8^{\circ}\right)$ from $H_{0}$, which is the field value where resonance would occur in the absence of fine structure. Finally, the amplitudes of the forbidden $\Delta M_{S}= \pm 1, \Delta M_{I}= \pm 1$ lines are related to $\left(D / H_{0}\right)^{2}$, while the shifts of their positions are proportional to $D^{2} / H_{0} \cdot{ }^{14,15}$

1. Hydrated MnKA Zeolites. (a) X-band Spectra. The room temperature X-band spectrum of a representative MnKA sample is given in Figure 1a. The central hyperfine sextet with $A=0.0089 \pm 0.0001 \mathrm{~cm}^{-1}$ is readily identified as the $M_{S}=$ $+1 / 2 \leftrightarrow-1 / 2$ transition $\left(\theta=90^{\circ}\right)$ of the $\mathrm{Mn}^{2+}$ ion. The two broad lines at lower field and the group of lines upfield of this $M_{S}=+1 / 2 \leftrightarrow-1 / 2$ sextet display only limited hyperfine structure at $298 \mathrm{~K}$. Cooling of the sample to $7 \mathrm{~K}$ left the overall spectral features unaltered. This confirms that the spectrum originates from a monomeric $\mathrm{Mn}^{2+}$ species. ${ }^{16}$ An observation at $333 \mathrm{~K}$, however, reveals a better resolved hyperfine structure (Figure 1b). The shape of the line pattern at $333 \mathrm{~K}$ suggests the presence of $\mathrm{Mn}^{2+}$ in an axially distorted environment. By numerical simulation of a corresponding powder pattern, stick spectra were generated for the positions of the fine and hyperfine structure lines and these are included in Figure 1b. Four of the five $\theta=$ $90^{\circ}$ fine lines, with 6-fold hyperfine splitting, are easily identified and were labeled as 1, 2, 3, and 5. The lack of resolution around $3800 \mathrm{G}$, where the fifth sextet is expected, is due to the overlap between the remaining $\theta=90^{\circ}$ sextet, labeled as $\mathbf{4}$, and the $\theta=41.8^{\circ}$ absorption maximum of the $M_{S}=+1 / 2$ $\leftrightarrow-1 / 2$ transition, labeled as $\mathbf{3}^{\prime}$. From such simulations, $D$ was estimated to amount to $0.035 \pm 0.002 \mathrm{~cm}^{-1}$. Note that the central $\theta=90^{\circ}$ sextet is centered around a $g_{\text {eff }}=2.052$. Compared to hydrated $\mathrm{Mn}^{2+}$ in faujasites, this represents a downfield shift of about $80 \mathrm{G}$, in agreement with the calculated shift $\left(-2 D^{2} / H_{0}\right){ }^{11}$ This axially distorted $\mathrm{Mn}^{2+}$ species in the KA zeolite is labeled as species $\mathbf{I}$.

(11) Woltermann, G. M.; Wasson, J. R. Inorg. Chem. 1973, 12, 2366.

(12) Shepherd, R. A.; Graham, W. R. M. J. Chem. Phys. 1984, 81, 6080.

(13) Tikhomirova, N. N.; Dobryakov, S. N.; Nikolaeva, I. V. Phys. Status Solidi A 1972, 10, 593.

(14) De Wijn, H. W.; Van Balderen, R. F. J. Chem. Phys. 1967, 46, 1381.

(15) Shaffer, J. S.; Farach, H. A.; Poole, C. P. Phys. Rev. B 1976, 13, 1869.

(16) Khangulov, S. V.; Pessiki, P. J.; Barynin, V. V.; Ash, D.; Dismukes, G. C. Biochemistry 1995, 34, 2015. 

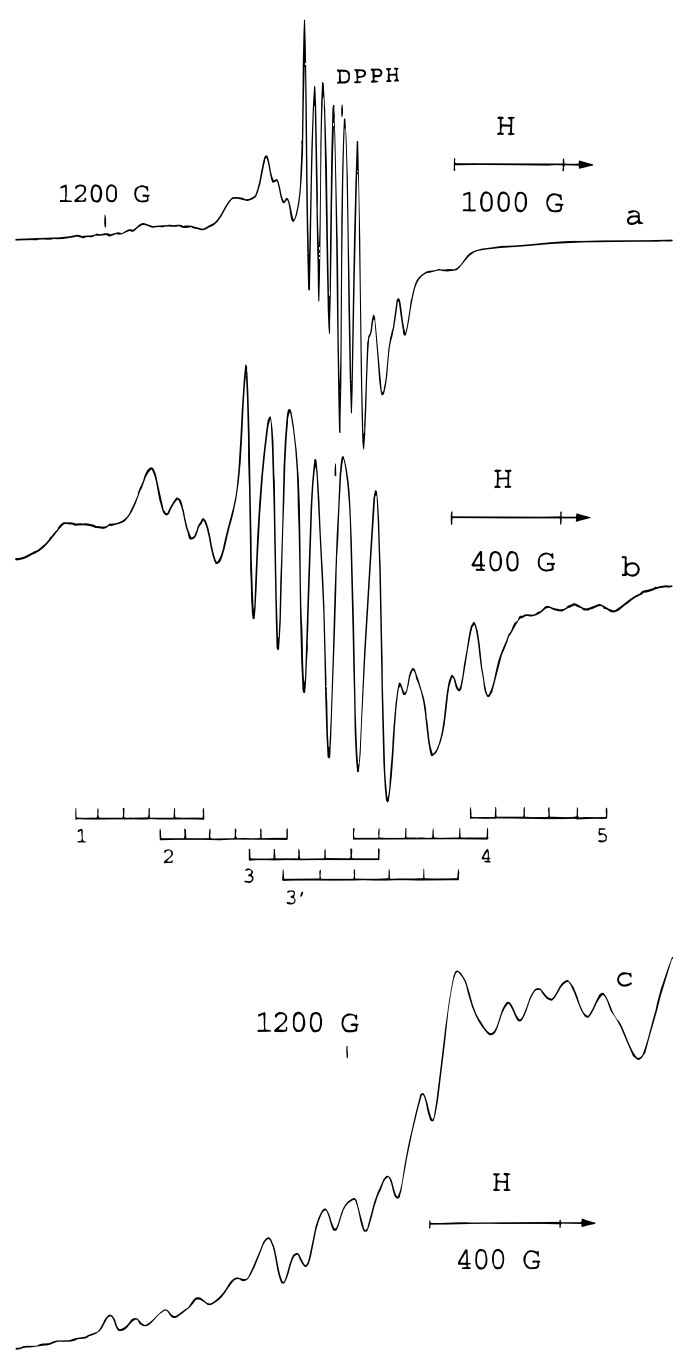

Figure 1. X-band ESR spectra of hydrated MnKA zeolite (0.1 wt \% Mn): (a and c) $298 \mathrm{~K}$, (b) $333 \mathrm{~K}$. For the stick diagram in b, positions were calculated with $D=0.035 \mathrm{~cm}^{-1}$ and $A=0.0089 \mathrm{~cm}^{-1}$. Labels for b are as follows: 1 and $5, M_{S}= \pm 5 / 2 \leftrightarrow \pm 3 / 2, \theta=90^{\circ} ; 2$ and $4, M_{S}$ $= \pm 3 / 2 \leftrightarrow \pm^{1} / 2, \theta=90^{\circ}: 3, M_{S}=+1 / 2 \leftrightarrow-1 / 2, \theta=90^{\circ} ; 3^{\prime}, M_{S}=+1 / 2$ $\leftrightarrow-1 / 2, \theta=41.8^{\circ}$.

Cooling of the samples invariably resulted in a broadening of the hyperfine lines or, for the non-central fine lines, even loss of hyperfine resolution. For $\mathrm{Mn}^{2+}$ ions in various solvents, line broadening upon temperature decrease has been attributed to a slower Brownian rotation of the complex as a whole, or to the slower fluctuations in the coordination sphere geometry. ${ }^{17}$ Cooling of the hydrated zeolite is expected to slow or ultimately freeze molecular motions in the proximity of the Mn sites. As a result, the fluctuations of the complex geometry become static, which leads to a distribution of the crystal-field parameters. It has been pointed out by Feher and by Dowsing and Ingram that especially the non-central lines of the fine structure are broadened by a distribution of the ZFS parameters, while the central transition is only slightly affected. ${ }^{18}$

Nevertheless, a clear trend can be discerned in the spectra of species $\mathbf{I}$ as the temperature is lowered. The non-central fine lines shift further away from $g=2$. From this variation, a gradual increase of the axial zero field splitting parameter can

(17) (a) Burlamacchi, L. J. Chem. Phys. 1971, 55, 1205. (b) Burlamacchi, L. Gazz. Chim. Ital. 1976, 106, 347. (c) Garrett, B. B.; Morgan, L. O. J. Chem. Phys. 1966, 44, 890.

(18) (a) Feher, E. R. Phys. Rev. 1964, 136, A145. (b) Dowsing, R. D.; Ingram, D. J. E. J. Magn. Reson. 1969, 1, 517.

(19) Cordischi, D.; Nelson, R. L.; Tench, A. J. Trans. Faraday Soc. 1969, 65,2740 .

$$
\left(H_{0}-H\right)(G)
$$

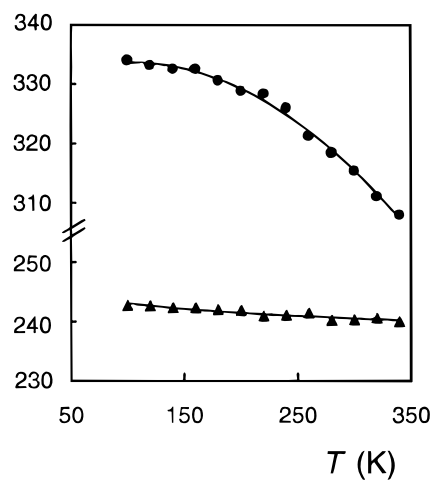

Figure 2. Temperature dependence of the position of the $\theta=90^{\circ}$ absorption maximum of the central $M_{S}=+1 / 2 \leftrightarrow-1 / 2$ transition of species I (in MnKA, ๑) and species II (in MnNaA, $\mathbf{\Delta}$ ). Positions are expressed as downfield shifts $\left(H_{0}-H, \mathrm{G}\right)$ from the DPPH resonance $\left(H_{0}\right)$, and were measured at the most downfield hyperfine line of the sextet $(H)$. For both species, $A=0.0089 \mathrm{~cm}^{-1}$ at all temperatures; hence the shifts for species $\mathbf{I}$ are due to a changing $D$ value.

be inferred, bringing $D$ to $0.039 \pm 0.002 \mathrm{~cm}^{-1}$ at temperatures below $140 \mathrm{~K}$. Meanwhile, the position of the central sextet varies with $T$, as plotted in Figure 2. At low temperature, this sextet is centered at $g_{\text {eff }}=2.068$, confirming the marked increase of the $D$ parameter.

A series of low-intensity lines is observed in the low-field domain between 400 and $2200 \mathrm{G}$, where eventually the $\Delta M_{S}=$ $\pm 1, \theta=90^{\circ}$ lines become dominant (Figure 1c). Several Mn hyperfine sextets are well resolved. Two types of lines might contribute to the absorption in this domain. First, the $\theta=0^{\circ}$ absorption edges of the $\Delta M_{S}= \pm 1$ transitions are spread around $g=2$ over a range approximately twice as broad as encompassed by the $\theta=90^{\circ}$ absorption maxima. However, some of the observed sextets extend much more downfield. It is therefore probable that at least part of these lines stem from the fine and hyperfine structure of the forbidden $\Delta M_{S}= \pm 2$ transitions. For $D=0.035 \mathrm{~cm}^{-1}$ and at X-band, the integrated intensity of these forbidden transitions is expected to be about $4 \%$ of that of the allowed $\Delta M_{S}= \pm 1$ transitions. ${ }^{14}$ However, it proved difficult to sort these lines into the expected four sextets, the zero-field splitting being relatively large in comparison with the X-band Zeeman energy.

(b) Q-band Spectra. Q-band measurements were undertaken to check the values of the splitting parameters estimated from the X-band experiments and a typical spectrum is given in Figure 3a. As the second-order shifts of the positions of the fine lines are inversely proportional to $H_{0}$, they are much smaller at Q-band frequency. ${ }^{11}$ As a result, a highly precise simulation of the line positions could be obtained, predicting the resonance positions within $4 \mathrm{G}$, i.e. the error on the field determination. $D$ was determined at $0.0356 \pm 0.0004 \mathrm{~cm}^{-1}$ at room temperature. At high Mn concentrations, two broad shoulders are observed around the main $\theta=90^{\circ}$ absorptions (Figure $3 \mathrm{~b}$ ). These features find their origin in the $\theta=0^{\circ}$ absorption edges of the allowed $M_{S}= \pm 1$ transitions. As the lower field limit of these $\theta=0^{\circ}$ lines is about $10000 \mathrm{G}$, these lines are well separated at Q-band frequency from possible half-field transitions.

The ratio of the intensities of the half-field $\Delta M_{S}= \pm 2$ lines relative to the $\Delta M_{S}= \pm 1$ lines is proportional to $\left(D / H_{0}\right)^{2}$. Halffield transitions are therefore about 14 times weaker at Q-band than at X-band. At 298 and at $100 \mathrm{~K}$, a broad line can be distinguished at $6300 \mathrm{G}$, which agrees well with $H_{0} / 2$ (Figure $3 c)$. This envelope is expected to contain four groups of lines, 


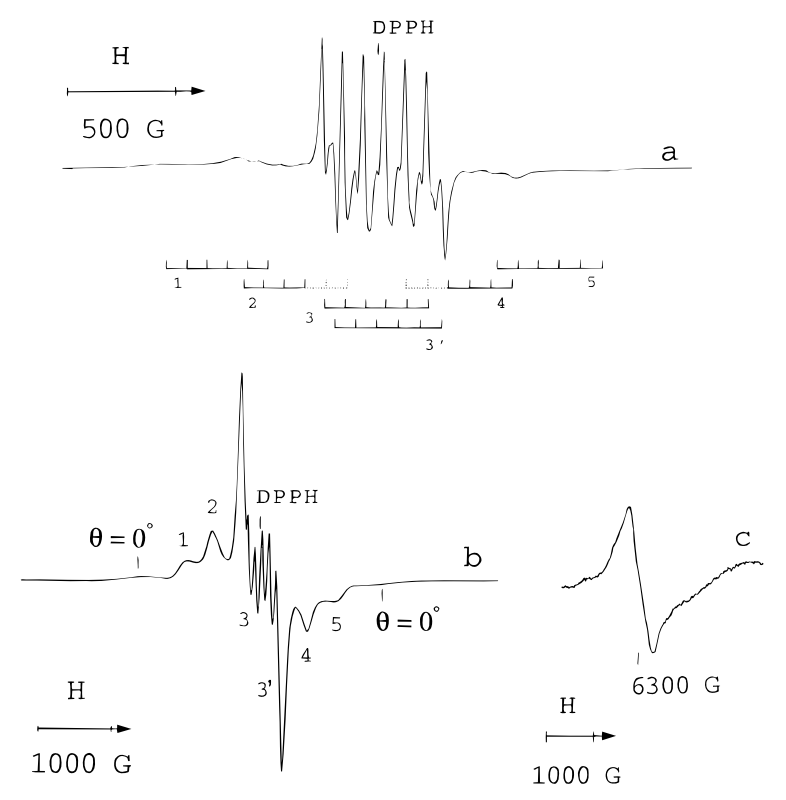

Figure 3. Q-band ESR spectra of hydrated MnKA zeolites: (a) 0.1 wt \%, $298 \mathrm{~K}$, (b, c) $1.7 \mathrm{wt} \%, 298 \mathrm{~K}$. The positions of the stick diagram in a were calculated with $D=0.0356 \mathrm{~cm}^{-1}$ and $A=0.0089 \mathrm{~cm}^{-1}$. Labels for $\mathrm{a}$ and $\mathrm{b}$ are as follows: 1 and $5, M_{S}= \pm 5 / 2 \leftrightarrow \pm \frac{3}{2}, \theta=$ $90^{\circ} ; 2$ and $4, M_{S}= \pm 3 / 2 \leftrightarrow \pm 1 / 2, \theta=90^{\circ} ; 3, M_{S}=+1 / 2 \leftrightarrow-1 / 2, \theta=$ $90^{\circ} ; 3^{\prime}, M_{S}=+1 / 2 \leftrightarrow-1 / 2, \theta=41.8^{\circ}$. The markers in $\mathrm{b}$ indicate the $\theta=0^{\circ}$ shoulders of the $\Delta M_{S}= \pm 1$ transitions. Spectrum c shows the half-field transition.

separated in first order by $D$; the two inner groups of fine lines should be twice as intense as the two outer groups. ${ }^{10}$ This corresponds well to the overall appearance of the spectrum, with a peak-to-peak width of $540 \mathrm{G}$.

2. Hydrated MnNaA Zeolites. (a) X-band Spectra. In the spectrum of hydrated MnNaA, two groups of lines can be distinguished. First, a sextet dominates the $\mathrm{X}$-band spectrum of Figure 4a. The center of this sextet with $A=0.0089 \mathrm{~cm}^{-1}$ is situated at $g_{\text {eff }}=2.006$, which indicates that for this species, the zero-field splitting must be much smaller than for species I, which dominated the spectrum in the case of MnKA. We propose this $\mathrm{Mn}$ species II to be an almost octahedral Mn complex. The non-central fine transitions were not observed for this species. At the high-field end of the central sextet, a small splitting, between 25 and $35 \mathrm{G}$, is visible (Figure 4c). It is reasonable to assume that these two lines belong to the $\theta=$ $90^{\circ}$ and $41.8^{\circ}$ sextets. Following the approach of Cordischi et al.,$^{19}$ a $D$ value of $0.010 \pm 0.001 \mathrm{~cm}^{-1}$ is obtained.

Apart from this species II, several other lines are observed in the spectrum, e.g. at 1100, 1900, 2800, and $4800 \mathrm{G}$, assigned to a species III (see below, Q-band data). Because they are spread over a broad field range, identification of these lines against the background may be difficult at low Mn concentrations, but by varying the Mn concentration, the relation between growing intensity of these lines and increasing Mn content was firmly established (Figure 4, a vs b). As the apparent splittings in this spectrum are of the same order as the X-band Zeeman energy, it was impossible to obtain the ZFS parameters from the X-band data alone.

(b) Q-band Spectra. The Q-band spectra of the same zeolite allowed us to assign these supplementary spectral lines to a species III. The zero-field splitting of this species III is dominated by a very large $D$ value of $0.14 \pm 0.01 \mathrm{~cm}^{-1}$ (Figure $5 \mathrm{~b})$. The groups of fine structure lines have been labeled on the spectrum by means of a calculated stick spectrum. With such a severe distortion of the Mn coordination, even small

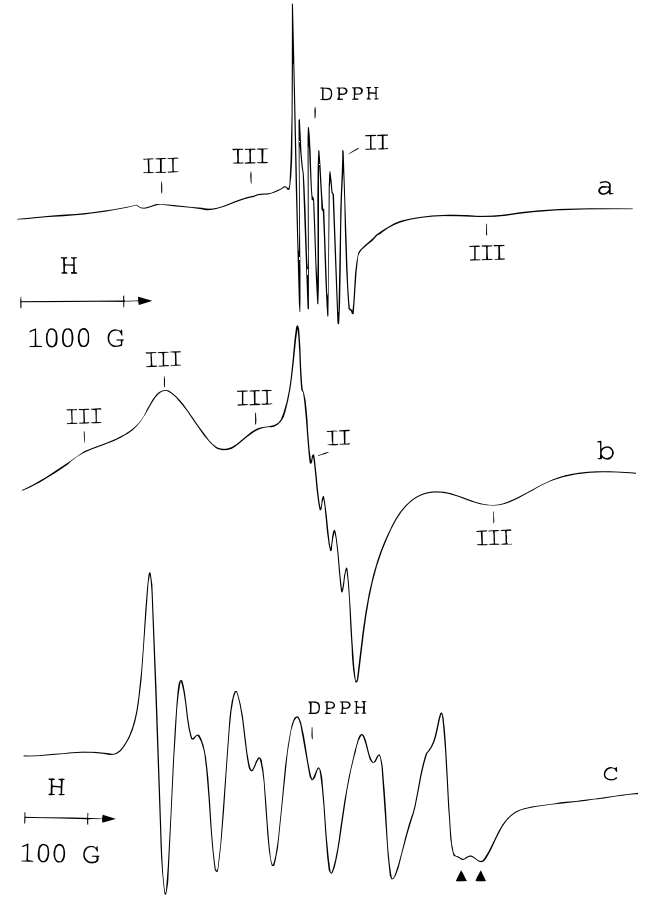

Figure 4. X-band ESR spectra of hydrated MnNaA zeolites, showing lines of species II and III: (a and c) $0.1 \mathrm{wt} \%, 298 \mathrm{~K}$, (b) $1.7 \mathrm{wt} \%$, $298 \mathrm{~K}$. In c the $\boldsymbol{\Delta}$ symbols indicate the splitting between the $\theta=90^{\circ}$ and $41.8^{\circ}$ sextets for $M_{S}=+1 / 2 \leftrightarrow-1 / 2$ in species II.

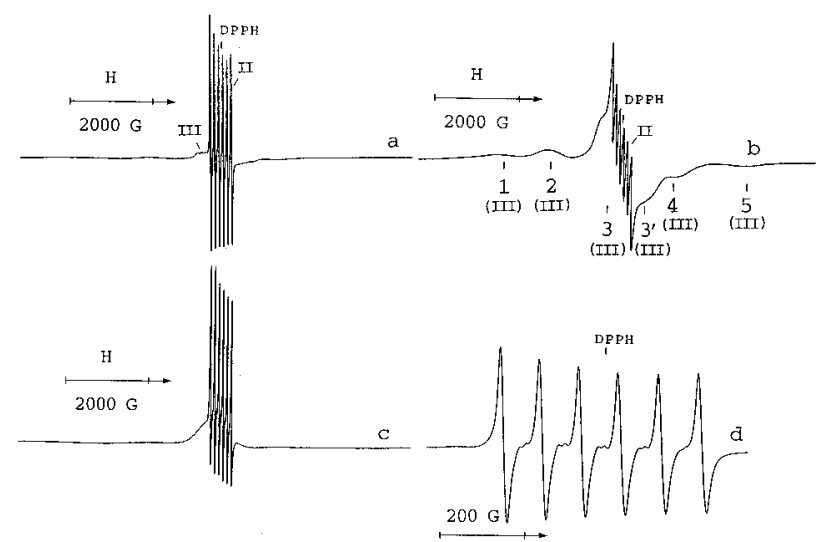

Figure 5. Q-band ESR spectra of hydrated MnNaA zeolites: (a and d) $0.1 \mathrm{wt} \%, 298 \mathrm{~K}$, (b) $1.7 \mathrm{wt} \%, 298 \mathrm{~K}$, (c) $0.1 \mathrm{wt} \%, 100 \mathrm{~K}$. Note in a the superimposed sextets of species II and species III, about 350 $\mathrm{G}$ upfield. The positions in the stick diagram for species III in b were calculated with $D=0.14 \mathrm{~cm}^{-1}$. Labels on the stick diagram as in Figure 3. Note the forbidden $\Delta M_{S}= \pm 1, \Delta M_{I}= \pm 1$ lines for species II in d.

deviations from the average $D$ value become significant in comparison with the hyperfine constant $A$. As a result, hardly any hyperfine structure can be observed for III. Only at 298 $\mathrm{K}$ and low Mn concentration, the $M_{S}=+1 / 2 \leftrightarrow-1 / 2, \theta=90^{\circ}$ sextets of II and III are observed simultaneously (Figure 5a). The sextet of III, with $A=0.0088 \pm 0.0002 \mathrm{~cm}^{-1}$, is centered $350 \mathrm{G}$ downfield from that of II. With $D_{\text {II }}$ smaller than 0.01 $\mathrm{cm}^{-1}, D_{\text {III }}$ is estimated to amount to $0.139 \mathrm{~cm}^{-1}$, confirming the value from the stick spectrum in Figure $5 \mathrm{~b}$. Within the resolution of our data, there is no evidence for $E$ being different from 0 . Hence III is described as a strongly axially distorted $\mathrm{Mn}^{2+}$ complex.

As with species I in MnKA, cooling of MnNaA resulted in a resolution loss for species III. With $\mathrm{MnNaA}(0.1 \%)$ at 100 $\mathrm{K}$, even the hyperfine structure of the central sextet of species III is lost (Figure 5c). 

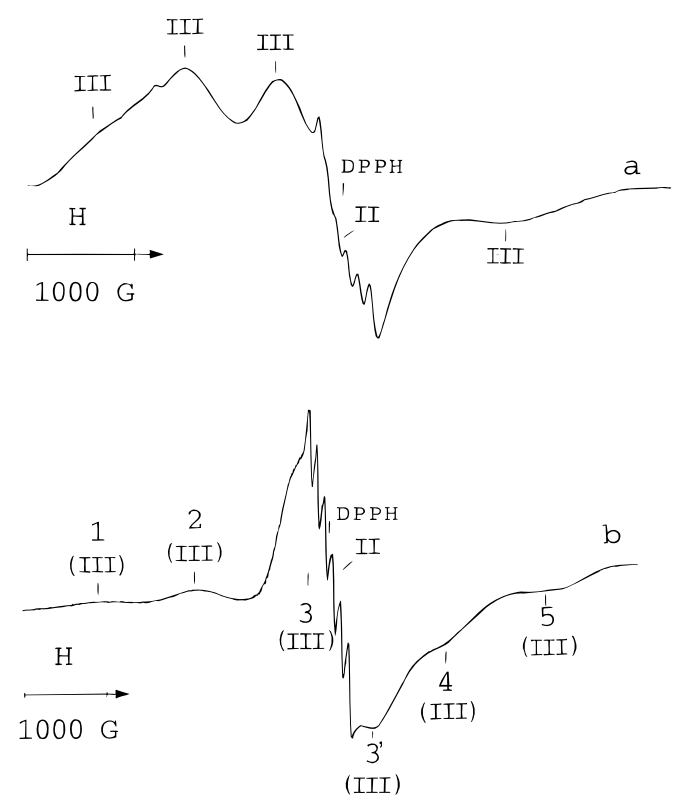

Figure 6. ESR spectra of $0.1 \mathrm{wt} \%$ hydrated MnLiA zeolite at $298 \mathrm{~K}$, showing lines of species II and III: (a) X-band (9.44 GHz), (b) Q-band $(35.0 \mathrm{GHz})$. Labels on $\mathrm{b}$ as in Figure 3.

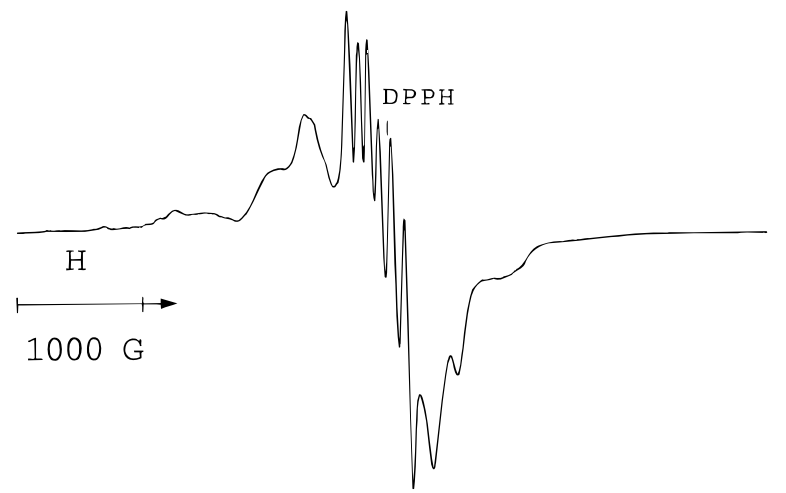

Figure 7. X-band ESR spectrum of hydrated MnCsNaA zeolite $(0.1$ wt \% Mn) at $298 \mathrm{~K}$.

The Q-band spectrum also yields a supplementary estimate for $D$ in species II. At $298 \mathrm{~K}$ and low Mn concentration, the forbidden $\Delta M_{S}= \pm 1, \Delta M_{I}= \pm 1$ lines of species II are resolved (Figure 5d). Their amplitude varies between 1 and $2 \%$ of the amplitude of the allowed $\Delta M_{S}= \pm 1, \Delta M_{I}=0$ lines. Following the approach of Poole et al., ${ }^{15} D_{\mathrm{II}}=0.009 \pm 0.002 \mathrm{~cm}^{-1}$ is obtained, in reasonable agreement with the previous estimate from the X-band spectrum (Figure $4 \mathrm{c}$ ).

3. Hydrated MnLiA Zeolites. In the spectra of $\mathrm{Mn}^{2+}$ exchanged LiA, recorded at 298 or $100 \mathrm{~K}$, the same species are detected as in the case of $\mathrm{MnNaA}$. The sextet, centered at $g_{\text {eff }}=2.006$, is ascribed to II, an almost octahedral $\mathrm{Mn}^{2+}$ species. All lines belonging to species III were also identified, in X- as well as in Q-band (Figure 6). However, the relative intensities of the signals of II and III are different in the $\mathrm{Na}^{+}$ and the $\mathrm{Li}^{+}$zeolites. In $\mathrm{NaA}$, the signal of species $\mathbf{I I}$ is relatively much stronger than that in LiA, where species III dominates the spectrum. Quantification of these differences in terms of site population is however difficult in view of the very different field range spanned by II and III.

4. Hydrated MnCsKA, MnCsNaA, and $\mathrm{MnNH}_{4} \mathrm{~A}$ Zeolites. The overall aspect of the spectra of these samples is very close to that observed for MnKA (Figure 7). Only species I, with $D$ between 0.035 and $0.039 \mathrm{~cm}^{-1}$, is identified. The dependence of $D$ on temperature is the same as in MnKA: $D$ increases with decreasing temperature.

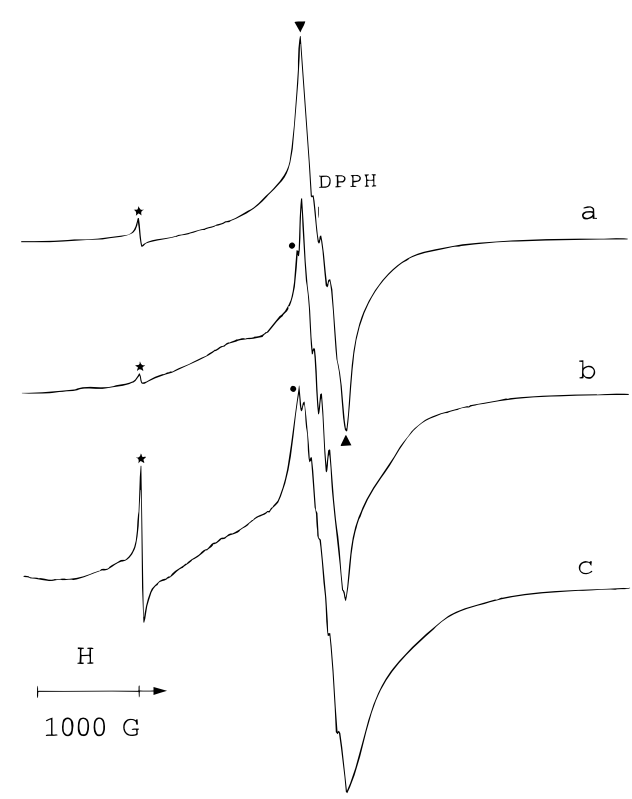

Figure 8. X-band spectra of fully dehydrated $0.025 \mathrm{wt} \% \mathrm{Mn} \mathrm{A}$ zeolites: (a) MnNaA, $298 \mathrm{~K}$, (b) MnKA, $298 \mathrm{~K}$, (c) MnNaA, $100 \mathrm{~K}$. The peak-to-peak widths were calculated as indicated by triangles $(\mathbf{\Lambda})$. Dots (-) mark the supplementary line, downfield of the maximum, in MnKA (b) and cooled MnNaA (c). * $*$ Fe impurities in the zeolite.

5. Dehydrated Zeolites. Dehydration at $673 \mathrm{~K}$ produces dramatic changes in the ESR spectra of MnNaA, MnKA, and MnLiA. In general, the spectra are less well defined than for the hydrated zeolites, and the presence of overlapping sextets close to $g=2$, even at very low Mn content, indicates that the speciation is not always uniform.

(a) MnKA and MnNaA. The spectra of dehydrated MnKA and $\mathrm{MnNaA}$ are dominated by the central transition of the fine structure. At room temperature, the peak-to-peak width (calculated as labeled in Figure 8a) amounts to $400 \pm 4 \mathrm{G}$, from which a maximum value $A=0.0070 \mathrm{~cm}^{-1}$ is obtained. In MnKA, an additional peak is observed downfield of the maximum in the first derivative spectrum (Figure $8 \mathrm{~b}$ ). This peak is also detected for $\mathrm{MnNaA}$ when the spectrum is recorded at $100 \mathrm{~K}$ (Figure 8c). The temperature-dependent spectral changes are fully reversible and indicate that the speciation fluctuates with temperature. The main, central transition is for both zeolites surrounded by wings, which are broader at lower temperatures. Such wings are typical for a distribution of the zero-field splitting parameters, and have been described for $\mathrm{Mn}^{2+}$ in glass environments, ${ }^{14,20}$ or for frozen solutions of $\mathrm{Mn}^{2+}$ phosphate complexes. ${ }^{17 \mathrm{~b}}$ We label the overall appearance of Mn in these zeolites as species IV, characterized by (i) a small hyperfine constant, (ii) overlapping sextets near $g=2$, and (iii) wings around the central absorption. It is stressed that species IV is a collective name for a distribution of similar Mn sites rather than a well-defined Mn complex.

(b) MnLiA. The X-band spectra of dehydrated MnLiA (Figure 9) display a line pattern which is recognized as that of an axially symmetric $\mathrm{Mn}$ ion, based on the analogy with the spectra in Figures $1 \mathrm{a}$ and $5 \mathrm{~b}$. The fine structure groups have been labeled on the spectra. From the positions of the noncentral fine lines, $D$ is estimated at $0.032 \pm 0.003 \mathrm{~cm}^{-1}$ at room temperature. There is no evidence for $E$ being different from zero. There is a remarkable and reversible dependence of the spectrum on temperature (Figure 9b). At $100 \mathrm{~K}, D$ has increased by about $60 \%$, to $0.050 \pm 0.003 \mathrm{~cm}^{-1}$. This species is denoted as species $\mathbf{V}$. There seem to be small amounts of

(20) Griscom, D. L.; Griscom, R. E. J. Chem. Phys. 1967, 47, 2711. 


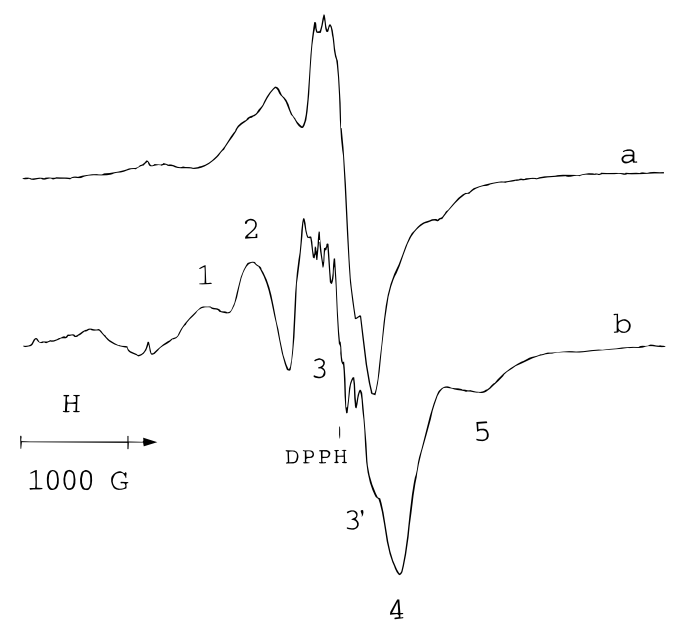

Figure 9. X-band spectra of fully dehydrated $0.025 \mathrm{wt} \% \mathrm{MnLiA}$ zeolite, recorded at (a) 298 and (b) $100 \mathrm{~K}: 1$ and 5, $M_{S}= \pm 5 / 2 \leftrightarrow$ $\pm^{3} / 2, \theta=90^{\circ} ; 2$ and $4, M_{S}= \pm^{3} / 2 \leftrightarrow \pm^{1 / 2}, \theta=90^{\circ} ; 3, M_{S}=+1 / 2 \leftrightarrow$ $-1 / 2, \theta=90^{\circ} ; 3^{\prime}, M_{S}=+1 / 2 \leftrightarrow-1 / 2, \theta=41.8^{\circ}$.

other species, which prevent precise observation of the hyperfine structure around $g=2$, and determination of $A$. In the lowfield domain, a complex pattern with several Mn hyperfine sextets is observed. As for species I, these lines can be related to the forbidden $\Delta M_{S}= \pm 2$ transitions.

6. Rehydration of Zeolites. Even a very short exposure of the dehydrated zeolites to water causes a spectral change. After 5 min of rehydration, $\mathrm{MnLiA}, \mathrm{MnNaA}$, and MnKA display spectra as shown in Figure 10, parts a and b. A sextet with $A$ $=0.0081 \mathrm{~cm}^{-1}$, at $g_{\text {eff }}=2.003$, is surrounded by wings. This species is labeled VI.

Further rehydration gradually and fully restores species $\mathbf{I}$ on MnKA. For MnNaA and MnLiA, species III is the first to reappear (Figure 10c). Only after extended water exposure is species II regenerated, but even then, the ratio II/III clearly remains lower in the rehydrated samples than before the dehydration.

\section{Discussion}

Table 1 gives an overview of the different $\mathrm{Mn}^{2+}$ species and their $A$ and $D$ parameters. Table 2 summarizes the dehydration-rehydration cycles for MnLiA, MnNaA, and MnKA.

Hydrated Zeolites. The overall spectroscopic picture allows us to divide the monovalent cations into two major groups. With $\mathrm{K}^{+}, \mathrm{Cs}^{+}$, and $\mathrm{NH}_{4}{ }^{+}$, species $\mathbf{I}$ is dominant. With $\mathrm{Na}^{+}$and $\mathrm{Li}^{+}$, species II and III occur simultaneously, though in varying concentrations. The grouping of these cations basically corresponds to their size. The studies of Kevan on the spectroscopy of $\mathrm{Cu}^{2+}$ exchanged $\mathrm{A}$ zeolites have led to a very similar conclusion. ${ }^{21-25}$ Our observations are therefore in excellent agreement with one of Kevan's major statements, namely that in A zeolites, exchanged with large monovalent cations, steric crowding makes it impossible to accommodate the regular, hexacoordinate hydrated transition metal ions. Thus steric arguments seem to prevail in the cation siting of both $\mathrm{Cu}$ and Mn A zeolites. This contrasts with the situation in $\mathrm{X}$ zeolites, where it was demonstrated both for $\mathrm{Cu}$ and for $\mathrm{Mn}$ that the

(21) Narayana, M.; Kevan, L. J. Chem. Phys. 1981, 75, 3269.

(22) Narayana, M.; Kevan, L. J. Phys. C: Solid State Phys. 1983, 16, 361 .

(23) Anderson, M. W.; Kevan, L. J. Phys. Chem. 1987, 91, 1850.

(24) Narayana, M.; Kevan, L. J. Chem. Soc., Faraday Trans. 1 1986, $82,213$.

(25) Ichikawa, T.; Kevan, L. J. Am. Chem. Soc. 1981, 103, 5355.

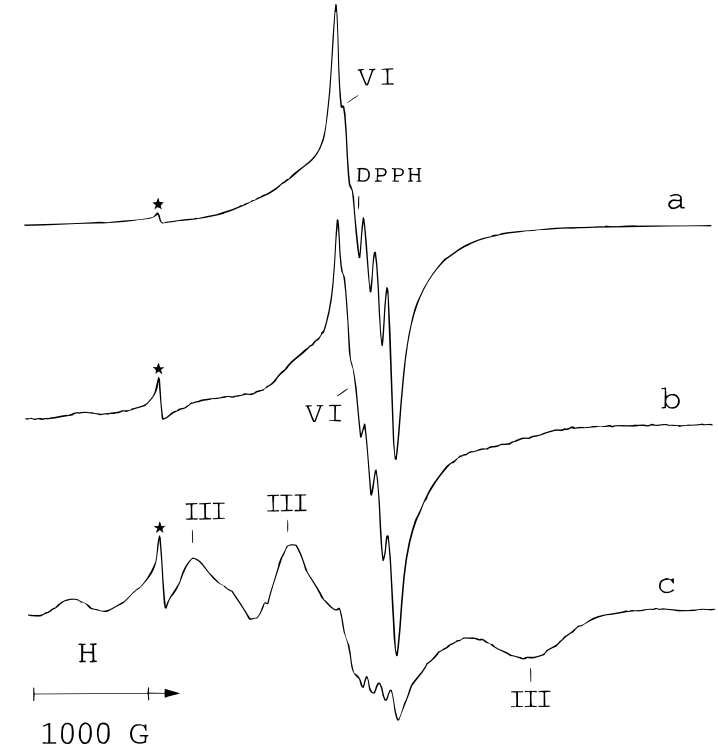

Figure 10. X-band spectra of partially rehydrated 0.025 wt \% Mn A zeolites, recorded at $298 \mathrm{~K}$ : (a) MnLiA, after 5 min of water exposure, (b) $\mathrm{MnNaA}$, after $5 \mathrm{~min}$ of water exposure, (c) $\mathrm{MnNaA}$, after $30 \mathrm{~min}$ of water exposure. Initially, only species VI is visible. After $30 \mathrm{~min}$, III dominates in MnNaA (compare Figure 10c with Figure 4a). * = Fe impurities in the zeolite.

Table 1. Spectroscopic Characteristics of $\mathrm{Mn}^{2+}$ Species in Zeolite $\mathrm{A}^{a}$

\begin{tabular}{lclll}
\hline & $|A|\left(\mathrm{cm}^{-1}\right)$ & $|D|\left(\mathrm{cm}^{-1}\right)$ & proposed coordination & \multicolumn{1}{c}{ co-cation } \\
\hline I & 0.0089 & $0.0356^{b}$ & distorted octahedral & $\mathrm{K}^{+}, \mathrm{Cs}^{+}, \mathrm{NH}_{4}^{+}$ \\
II & 0.0089 & 0.01 & octahedral & $\mathrm{Na}^{+}, \mathrm{Li}^{+}$ \\
III & 0.0088 & 0.14 & trigonal bipyramidal & $\mathrm{Na}^{+}, \mathrm{Li}^{+}$ \\
IV & $<0.0070$ & $c$ & see text & $\mathrm{Na}^{+}, \mathrm{K}^{+}$ \\
V & $c$ & $0.032^{b}$ & trigonal & $\mathrm{Li}^{+}$ \\
VI & 0.0081 & close to 0 & tetrahedral & $\mathrm{Na}^{+}, \mathrm{Li}^{+}, \mathrm{K}^{+}$ \\
\hline
\end{tabular}

${ }^{a}$ As expected, the $g$ values of the $\mathrm{Mn}^{2+}$ species were all in the range 2.001-2.004. Significant deviations of $g_{\text {eff }}$ from these values were always due to zero-field splitting. ${ }^{b}$ Value at $298 \mathrm{~K}$. ${ }^{c}$ Value not determined.

Table 2. Evolution of $\mathrm{Mn}^{2+}$ Species in A Zeolites with Different Monovalent Co-cations during a Dehydration-Rehydration Cycle

\begin{tabular}{lccc}
\hline \multicolumn{1}{c}{ co-cation } & $\mathrm{Li}^{+}$ & $\mathrm{Na}^{+}$ & $\mathrm{K}^{+}$ \\
\hline hydrated & III (II) & II (III) & I \\
dehydrated at $673 \mathrm{~K}$ & $\downarrow$ & $\downarrow$ & $\downarrow$ \\
after 5 min of $\mathrm{H}_{2} \mathrm{O}$ exposure & $\mathbf{V}$ & IV & IV \\
after 30 min of $\mathrm{H}_{2} \mathrm{O}$ exposure & VI & $\downarrow$ & $\downarrow$ \\
& III & III (II) & I \\
\hline
\end{tabular}

cation location is determined by the competitive hydration of the metal ion and its co-cation. ${ }^{2 a, 5}$

It is well-established that in hydrated $\mathrm{KA}, \mathrm{CaNaA}$, and $\mathrm{NH}_{4} \mathrm{~A}$ zeolites, $\mathrm{Cu}^{2+}$ assumes an unusual pseudotetrahedral $\mathrm{S}_{\mathrm{II}}{ }^{*}$ coordination, with the $\mathrm{Cu}^{2+}$ ion coordinated to three oxygen atoms of a 6-ring window of a sodalite cage, and with a water molecule as the fourth ligand. ${ }^{22-24}$ In the case of $\mathrm{Mn}^{2+}$, a tetrahedral coordination would be characterized by a decreased value of the hyperfine coupling constant $A .{ }^{26}$ The $A$ value of $0.0089 \mathrm{~cm}^{-1}$, observed in $\mathrm{KA}, \mathrm{NH}_{4} \mathrm{~A}$, and the Cs zeolites, therefore rules out such a coordination. Nevertheless, the substantial deviation of the $D$ value from 0 indicates that the

(26) There is a correlation between the value of the hyperfine coupling constant $A$ and the coordination number of $\mathrm{Mn}^{2+}$ : Abragam, A.; Bleaney, B. Electron Paramagnetic Resonance of Transition Ions; Clarendon Press, Oxford, 1970; p 440. 
effect of the crowding on the Mn coordination sphere is strong, leading to an axially symmetric 6- or possibly 5-fold Mn coordination. A possible location for $\mathrm{Mn}^{2+}$ species I would be a pseudooctahedral $\mathrm{S}_{\mathrm{II}}$ * position, in which $\mathrm{Mn}^{2+}$ would be coordinated to three lattice oxygens and to three water molecules, resulting in an imperfect antiprism, in which axial symmetry is maintained.

Hydrated $\mathrm{NaA}$ and LiA zeolites are much less sterically crowded, and it is not surprising that in these zeolites $\mathrm{Mn}^{2+}$ can assume a nearly octahedral coordination in species II. This species II may be a free $\mathrm{Mn}\left(\mathrm{H}_{2} \mathrm{O}\right)_{6}{ }^{2+}$ complex, as in $\mathrm{NaX}$ and NaY zeolites. ${ }^{5}$ Species III is characterized by a substantially large value of $D\left(0.14 \mathrm{~cm}^{-1}\right)$. At first sight it is difficult to understand why in $\mathrm{NaA}$ and $\mathrm{LiA}$, with less steric crowding, the distortion of the $\mathrm{Mn}^{2+}$ coordination sphere is even more severe than in $\mathrm{KA}$ or $\mathrm{NH}_{4} \mathrm{~A}$. However, for the case of $\mathrm{MnNaA}$, $\mathrm{X}$-ray diffraction data are available for evaluation of the $\mathrm{Mn}^{2+}$ siting. For a hydrated $\mathrm{NaA}$ zeolite, exchanged to a high degree with $\mathrm{Mn}^{2+}$, Seff et al. found $\mathrm{Mn}^{2+}$ to be located mainly in site $\mathrm{S}_{\mathrm{II}}$, where it assumes a trigonal-bipyramidal coordination. ${ }^{27}$ Three lattice oxygens of a 6-ring constitute the equatorial plane of the trigonal bipyramid, while two water molecules are located at its top and bottom, one inside the sodalite cage and one outside. Such a siting appears to match the coordination requirements derived from the parameters of species III. For an axially symmetric Mn trigonal bipyramid, a large $D$ and a negligible $E$ have indeed been reported. ${ }^{28}$ The bond lengths reported by Seff $\left(\mathrm{Mn}-\mathrm{O}_{\text {lattice }}=2.28 \AA ; \mathrm{Mn}-\mathrm{O}_{\text {water }}=2.04 \AA\right)$ point toward a strong compression of the trigonal bipyramid, which is expected to result in a large $D$ value, with $E$ close to zero.

Dehydrated Zeolites. Species IV and V are both found in highly dehydrated A zeolites. While in faujasite structures dehydration often brings cations to a hexacoordinate position inside the hexagonal prisms, dehydrated A zeolites offer sites with a limited number of coordinating atoms to metal ions. An X-ray study by Seff et al. of $\mathrm{Mn}^{2+}$ in dehydrated $\mathrm{NaA}$ has suggested an $\mathrm{S}_{\text {II }}$ location for $\mathrm{Mn}^{2+}$, where the metal ion is only coordinated by three lattice oxygen atoms of the zeolite sixring. ${ }^{27}$ This 3 -fold coordination corresponds to a strong "axial elongation" of a trigonal bipyramid, by removal of the apical ligands. To our knowledge, the Mn ESR literature does not contain any species that is directly comparable to this situation, which makes it difficult to predict values for the ZFS parameters. However, in such a trigonal site, axial symmetry is preserved. This should lead to a large $D$, but to an $E$ value close to zero. ${ }^{28}$ The parameters of species $\mathbf{V}$ may well be compatible with such a coordination. Therefore it seems that in dehydrated MnLiA, $\mathrm{Mn}^{2+}$ resides in trigonal coordination at $\mathrm{S}_{\mathrm{II}}$. Our data are thus complementary to those of Klier, ${ }^{29}$ who has previously described 723.

(27) Yanagida, R. Y.; Vance, T. B.; Seff, K. Inorg. Chem. 1974, 13,

(28) Axially symmetric, trigonal bipyramidal complexes of $\mathrm{Mn}^{2+}$ are rare. To our knowledge, mainly the complexes with the protonated 1,4diazabicyclo[2.2.2] octane $\left(\mathrm{HL}^{+}\right)$have been studied. The ESR parameters of complexes of the types $\left[\mathrm{Mn}\left(\mathrm{HL}^{+}\right)_{2} \mathrm{X}_{3}\right] \mathrm{X}(\mathrm{X}=\mathrm{Cl}, \mathrm{Br})$ have been determined: Birdy, R. B.; Brun, G.; Goodgame, D. M. L.; Goodgame, M. J. Chem. Soc. Dalton Trans. 1979, 149. For these axially symmetric fivecoordinate complexes, $D$ varies between 0.31 and $0.6 \mathrm{~cm}^{-1} ; E / D \leq 0.03$. The values of $D$ for these complexes are considerably larger than what is observed for species V. However, it should be remembered that in the complexes of Goodgame et al., the trigonal bipyramid is subjected to an axial compression instead of an axial elongation. Moreover, the amine ligands in Goodgame's complexes are much stronger $\sigma$ donors than the oxygen atoms of a zeolite lattice. Therefore, a quantitative comparison of the ZFS parameters is not straightforward.

(29) (a) Klier, K.; Ralek, J. J. Phys. Chem. Solids 1968, 29, 951. (b) Klier, K. Adv. Chem. Ser. 1971, 101, 480. the unusual trigonal coordination of $\mathrm{Ni}^{2+}$ and $\mathrm{Co}^{2+}$ in dehydrated zeolite A by reflectance electronic spectroscopy.

The situation is less obvious for species IV. The low $A$ value and the dehydrated state of the zeolite exclude 6- or 5-fold coordination. ${ }^{26}$ Both 3 -fold and pseudotetrahedral coordination are possible, but the 4-fold coordination is less likely in view of the results of the rehydration experiment (see below). In contrast to $\mathbf{V}$, IV is a species not having a well-defined axial or rhombic distortion. Based on ESR data alone, it is therefore difficult to ascribe IV to a clearly defined lattice site.

Rehydrated Zeolites. Species VI is immediately formed upon water exposure of the coordinatively unsaturated species IV and V. The $A$ value of species VI $\left(0.0081 \mathrm{~cm}^{-1}\right)$ is intermediate between those of species I, II, and III (0.0089 $\left.\mathrm{cm}^{-1}\right)$ and species IV $\left(<0.0070 \mathrm{~cm}^{-1}\right)$. Species VI is clearly highly symmetric, e.g., close to octahedral or close to tetrahedral. The intermediate value of the $A$ parameter rather favors the latter possibility. The location of $\mathrm{Mn}$ in species VI may be for instance site $\mathrm{S}_{\mathrm{II}}$ *, with coordination to three oxygen atoms of the sodalite 6-ring and to one water molecule.

The order of appearance of the signals in MnNaA and MnLiA (first III, then II) is interesting. It suggests that III contains fewer water molecules in its coordination sphere than II. This is in full agreement with the proposals made above.

\section{Conclusions}

The nature of the different $\mathrm{Mn}^{2+}$ species and their interconversion proposed in this work are summarized in Tables 1 and 2. Of the six species presented, only species II (in hydrated $\mathrm{NaA}$ ), IV (in dehydrated $\mathrm{NaA}$ ), and VI (in partially hydrated $\mathrm{NaA}$ ) had been reported in a previous ESR study. ${ }^{6}$ The three species with the larger ZFS parameters (I, III, and V) were hitherto unknown. It seems therefore essential for a correct identification of Mn species to record ESR spectra at different frequencies and Mn concentrations. A similar approach has been applied recently in a re-investigation of the ZFS in organic $\mathrm{Mn}^{2+}$ complexes. ${ }^{30}$ Moreover, we have tried, wherever possible, to estimate the ZFS parameters from the spectra by at least two independent criteria.

Our results also demonstrate that zero-field splittings can be directly observed in the powder spectra of Mn zeolites, which contrasts with all previous studies. In some cases, the uniformity of the Mn sites has been demonstrated beyond doubt, e.g. for MnAPOs with low Mn content, where ESR has been combined with ESEM. ${ }^{7}$ In other studies, the presence of Mn in different coordination geometries, indicated by the observation of "wings" in the spectrum, may however have been underestimated. The observation of $\mathrm{Fe}$ in iron sodalite, where the sites are welldefined, and iron faujasite, with much more disorder, has led to similar caveat. ${ }^{4}$

The new species we propose (especially III and V) are similar to those found in related X-ray studies. Axial symmetry-whether in a trigonal bipyramid or in a planar 3-fold coordination-is very typical for transition metal sites in A zeolites, and our observations demonstrate that the $D$ values are remarkably large. The sensitivity of the ZFS parameters to even minute changes in coordination is beautifully illustrated in the fully reversible temperature dependence of the ZFS, as documented for IV, but especially for $\mathbf{I}$ and $\mathbf{V}$.

It is obvious that Mn siting in A zeolites is strongly affected by the co-cation present. This confirms the earlier observations for $\mathrm{Cu}^{2+}$ zeolites.

(30) Bryan Lynch, W.; Samuel Boorse, R.; Freed, J. H. J. Am. Chem. Soc. 1993, 115, 10909. 
Although this work defines major features of the Mn siting in zeolite A as observed with ESR, some details of the coordination would benefit from additional structural insight, for example regarding the number of water molecules around $\mathrm{Mn}$, or the interaction with various adsorbates. In this respect $\mathrm{X}$-ray diffraction or particularly electron spin echo envelope modulation (ESEEM) studies may be helpful.

Acknowledgment. This work was sponsored by the U.S. Department of Energy. We thank Professor M. W. McElfresh and Shi-Li (Purdue University) for performing SQUID experi- ments. We are mostly indebted to Professor R. Linn Belford and Dr. A. Smirnov (ESR Research Center, University of Illinois at Urbana-Champaign) for the use of their Q-band spectrometer. We thank Professor F. Mabbs and Dr. D. Collison (University of Manchester, U.K.) for recording the Q-spectrum in Figure 3a. B.M.W. thanks the Belgian National Fund for Scientific Research (N.F.W.O.) for a position as research assistant, and D.D.V. is a postdoctoral researcher of N.F.W.O.

JA960012D 